\title{
Recent results and prospects from NA62
}

\author{
Andrea Bizzeti ${ }^{1,2, \star}$ \\ ${ }^{1}$ Department of Physics, Informatics and Mathematics, University of Modena and Reggio Emilia, Italy \\ ${ }^{2}$ Istituto Nazionale di Fisica Nucleare - Sezione di Firenze, Italy
}

\begin{abstract}
A large sample of charged kaon decays in 2007 has been collected by the NA62 experiment at CERN SPS using the experimental setup of the former NA48 experiment. Its intense kaon beam provides an abundant source of tagged neutral pions in vacuum. A measurement of the electromagnetic transition form factor slope of the neutral pion from $1.05 \times 10^{6}$ fully reconstructed $\pi^{0}$ Dalitz decays is presented. The obtained preliminary value $a=\left(3.70 \pm 0.53_{\text {stat }} \pm 0.36_{\text {syst }}\right) \times 10^{-2}$ is the first $5.8 \sigma$ observation of a non-zero slope in the time-like region of momentum transfer.

$K^{+} \rightarrow \pi^{+} v \bar{v}$ is a theoretically very clean decay where indirect effects of new physics may be detectable. The NA62 apparatus has been significantly upgraded between 2008 and 2014 in order to measure the branching ratio of this decay with $10 \%$ precision. The NA62 experiment took data with the new setup in pilot runs in 2014 and 2015, reaching the design beam intensity. Results of first data quality studies in view of the 2016-2017 physics runs are presented.
\end{abstract}

\footnotetext{
${ }^{\star}$ for the NA62 Collaboration: G. Aglieri Rinella, R. Aliberti, F. Ambrosino, R. Ammendola, B. Angelucci, A. Antonelli, G. Anzivino, R. Arcidiacono, I. Azhinenko, S. Balev, M. Barbanera, J. Bendotti, A. Biagioni, L. Bician, C. Biino, A. Bizzeti, T. Blazek, A. Blik, B. Bloch-Devaux, V. Bolotov, V. Bonaiuto, M. Boretto, M. Bragadireanu, D. Britton, G. Britvich, M.B. Brunetti, D. Bryman, F. Bucci, F. Butin, E. Capitolo, C. Capoccia, T. Capussela, A. Cassese, A. Catinaccio, A. Cecchetti, A. Ceccucci, P. Cenci, V. Cerny, C. Cerri, B. Checcucci, O. Chikilev, S. Chiozzi, R. Ciaranfi, G. Collazuol, A. Conovaloff, P. Cooke, P. Cooper, G. Corradi, E. Cortina Gil, F. Costantini, F. Cotorobai, A. Cotta Ramusino, D. Coward, G. D'Agostini, J. Dainton, P. Dalpiaz, H. Danielsson, J. Degrange, N. De Simone, D. Di Filippo, L. Di Lella, S. Di Lorenzo, N. Dixon, N. Doble, B. Dobrich, V. Duk, V. Elsha, J. Engelfried, T. Enik, N. Estrada, V. Falaleev, R. Fantechi, V. Fascianelli, L. Federici, S. Fedotov, M. Fiorini, J. Fry, J. Fu, A. Fucci, L. Fulton, S. Gallorini, S. Galeotti, E. Gamberini, L. Gatignon, G. Georgiev, A. Gianoli, M. Giorgi, S. Giudici, L. Glonti, A. Goncalves Martins, F. Gonnella, E. Goudzovski, R. Guida, E. Gushchin, F. Hahn, B. Hallgren, H. Heath, F. Herman, T. Husek, O. Hutanu, D. Hutchcroft, L. Iacobuzio, E. Iacopini, E. Imbergamo, O. Jamet, P. Jarron, E. Jones, T. Jones K. Kampf, J. Kaplon, V. Kekelidze, S. Kholodenko, G. Khoriauli, A. Khotyantsev, A. Khudyakov, Yu. Kiryushin, A. Kleimenova, K. Kleinknecht, A. Kluge, M. Koval, V. Kozhuharov, M. Krivda, Z. Kucerova, Yu. Kudenko, J. Kunze, G. Lamanna, G. Latino, C. Lazzeroni, G. Lehmann-Miotto, R. Lenci, M. Lenti, E. Leonardi, P. Lichard, R. Lietava, L. Litov, R. Lollini, D. Lomidze, A. Lonardo, M. Lupi, N. Lurkin, K. McCormick, D. Madigozhin, G. Maire, C. Mandeiro, I. Mannelli, G. Mannocchi, A. Mapelli, F. Marchetto, R. Marchevski, S. Martellotti, P. Massarotti, K. Massri, P. Matak, E. Maurice, A. Mefodev, E. Menichetti, E. Minucci, M. Mirra, M. Misheva, N. Molokanova, J. Morant, M. Morel, M. Moulson, S. Movchan, D. Munday, M. Napolitano, I. Neri, F. Newson, A. Norton, M. Noy, G. Nuessle, T. Numao, V. Obraztsov, A. Ostankov, S. Padolski, R. Page, V. Palladino, G. Paoluzzi, C. Parkinson, E. Pedreschi, M. Pepe, F. Perez Gomez, M. Perrin-Terrin, L. Peruzzo, P. Petrov, F. Petrucci, R. Piandani, M. Piccini, D. Pietreanu, J. Pinzino, I. Polenkevich, L. Pontisso, Yu. Potrebenikov, D. Protopopescu, F. Raffaelli, M. Raggi, P. Riedler, A. Romano, P. Rubin, G. Ruggiero, V. Russo, V. Ryjov, A. Salamon, G. Salina, V. Samsonov, C. Santoni, G. Saracino, F. Sargeni, V. Semenov, A. Sergi, M. Serra, A. Shaikhiev, S. Shkarovskiy, I. Skillicorn, D. Soldi, A. Sotnikov, V. Sugonyaev, M. Sozzi, T. Spadaro, F. Spinella, R. Staley, A. Sturgess, P. Sutcliffe, N. Szilasi, D. Tagnani, S. Trilov, M. Valdata-Nappi, P. Valente, M. Vasile, T. Vassilieva, B. Velghe, M. Veltri, S. Venditti, P. Vicini, R. Volpe, M. Vormstein, H. Wahl, R. Wanke, P. Wertelaers, A. Winhart, R. Winston, B. Wrona, O. Yushchenko, M. Zamkovsky, A. Zinchenko.
} 


\section{The NA62 experiment}

NA62 is a fixed target experiment using a secondary hadron beam from SPS accelerator to perform flavor physics studies mainly in the charged kaon sector. NA62 collected data in 2007 using the same beam line and detecting apparatus of the earlier NA48 experiment[1], to study lepton universality in kaon decays through the measurement of the ratio between $K_{e 2}$ and $K_{\mu 2}$ leptonic decays[2] $R_{K}=$ $\Gamma\left(K^{ \pm} \rightarrow e^{ \pm} v\right) / \Gamma\left(K^{ \pm} \rightarrow \mu^{ \pm} v\right)$. After an R\&D and construction phase started in 2008, the experimental apparatus has been replaced by a new one designed to measure precisely the branching ratio of the $K^{+} \rightarrow \pi^{+} v \bar{v}$ decay. First data with the new NA62 setup have been collected in 2014/2015, mainly to commission detectors and perform data quality studies.

\section{Measurement of the $\pi^{0}$ transition form factor}

The neutral pion is the lightest meson and plays an important role in the study of low-energy properties of the strong interaction. The differential decay width of the subleading Dalitz decay $\pi^{0} \rightarrow \gamma e^{+} e^{-}\left(\pi_{D}^{0}\right)$ normalized to the leading decay $\pi^{0} \rightarrow \gamma \gamma\left(\pi_{\gamma \gamma}^{0}\right)$ as a function of the particle 4-momenta is given by:

$$
\frac{1}{\Gamma\left(\pi_{\gamma \gamma}^{0}\right)} \frac{\mathrm{d}^{2} \Gamma\left(\pi_{D}^{0}\right)}{\mathrm{d} x \mathrm{~d} y}=\frac{\alpha}{4 \pi} \frac{(1-x)^{3}}{x}\left(1+y^{2}+\frac{r^{2}}{x}\right)|\mathcal{F}(x)|^{2}[1+\delta(x, y)],
$$

where $x=\left(P_{e^{+}}+P_{e^{-}}\right)^{2} / m_{\pi^{0}}^{2}, y=2 P_{\pi^{0}} \cdot\left(P_{e^{+}}-P_{e^{-}}\right) /\left[m_{\pi^{0}}^{2}(1-x)\right], r^{2}=4 m_{e}^{2} / m_{\pi^{0}}^{2} \leq x \leq 1, \mathcal{F}(x)$ is the transition form factor (TFF) describing the hadronic physics in the $\gamma \gamma^{*}$ vertex and $\delta(x, y)$ contains the radiative corrections to the $\pi^{0}$ Dalitz decay. The TFF is usually parametrized as $\mathcal{F}(x)=1+a x$, where $a$ is called the TFF slope parameter. The TFF enters in the predictions of observable quantities like the rate of $\pi^{0} \rightarrow e^{+} e^{-}$decay and the anomalous magnetic moment of the muon. It has been extensively studied theoretically[3-8] and the slope parameter has been measured experimentally in the time-like[9-12] as well as in the space-like domain[13-16].

The charged kaons decaying in flight in the NA62 beam provide an abundant source of $\pi^{0} \mathrm{~s}$, mainly from the $K^{ \pm} \rightarrow \pi^{ \pm} \pi^{0}$ decay. NA62 collected about $2 \times 10^{10} K^{ \pm}$decays in 2007 , allowing the extraction of the slope parameter $a$ through the study of the $x$ spectrum of the $\pi_{D}^{0}$ decay, according to eq. 1 integrated over $y$. The radiative correction $\delta(x, y)$ has been studied extensively[17-20] and the most up-to-date calculation[20] has been implemented by NA62 in the Monte Carlo simulation of the $\pi_{D}^{0}$ decay.

\subsection{The NA62 setup in 2007 data taking}

The NA62 experiment detects in-flight decays of charged kaons at CERN SPS. The NA48 beam line and apparatus were used in NA62 2007 run, with different beam parameteres. The primary $400 \mathrm{GeV} / \mathrm{c}$ proton beam from CERN SPS impinged a beryllium target, producing charged particles, $6 \%$ of which were $K^{ \pm}$. A $100 \mathrm{~m}$ long beam line selected, focused and transported two simultaneous oppositely charged secondary beams of $(74.0 \pm 1.4) \mathrm{GeV} / \mathrm{c}$ momentum down to a $100 \mathrm{~m}$ long evacuated region. Particles produced by kaon decays were detected by the experimental apparatus located downstream. Charged particles were traced by a magnetic spectrometer consisting of a dipole magnet and four drift chamber stations. An array of horizontal and vertical plastic scintillator slabs provided fast signals for triggering and sub-ns timestamping of charged particles. The energy and position of photons and electrons were precisely measured by a quasi-homogeneous liquid krypton electromagnetic calorimeter (LKr). A muon detector (MUV) composed of iron absorbers and three planes of scintillator counters was used to identify muons. An iron-scintillator hadron calorimeter and several photon veto counters completed the experimental apparatus, a detailed description of which can be found in Ref. [1]. In 2007 run the trigger was optimized to select electron events by measuring the energy deposit in LKr. 


\subsection{Data analysis and preliminary results}

Events containing a $K^{ \pm} \rightarrow \pi^{+} \pi^{0}$ decays followed by the $\pi^{0} \rightarrow e^{+} e^{-} \gamma$ decay are selected by performing a full kinematic reconstruction. Three tracks in the magnetic spectrometer are required, coming from a common vertex inside the fiducial decay region. The photon is identified by a single energy deposit in $\mathrm{LKr}$, separated in space from the track impact points at the calorimeter surface. The total reconstructed momentum from photon and tracks has to be consistent with the nominal beam momentum magnitude and direction within the experimental resolution. Assuming the track with charge opposite to $K^{ \pm}$to be the $e^{\mp}$, events are selected if one and only one of the two possible $\left(e^{ \pm}, \pi^{ \pm}\right)$mass hypotheses for the two remaining tracks is compatible with the kinematics of the $K^{+} \rightarrow \pi_{D}^{0}$ decay within the resolution of the reconstructed $\pi^{0}$ and kaon mass. In order to be consistent with the simulation of the trigger conditions, at least one of the $e^{ \pm}$is required to have a momentum larger than $5.5 \mathrm{GeV} / \mathrm{c}$ and to deposit more than $80 \%$ of its energy in the LKr; moreover, the total energy detected in the LKr must not exceed $14 \mathrm{GeV}$. Due to the acceptance not being well reproduced in the simulation for events with low $x$, the signal region is defined as $x>0.01$, equivalent to $m_{e e}>13.5 \mathrm{MeV} / \mathrm{c}^{2}$.

About $1.05 \times 10^{6} \pi_{D}^{0}$ decays have been fully reconstructed. The TFF slope parameter $a$ is extracted by fitting the $x$ distribution of data and simulation, using equipopolous bins. The different hypotheses are tested by reweighting the MC events simulated with a known slope $a_{\text {sim }}=0.032$. The main contributions to the systematic uncertainty come from the simulation of the beam spectrum and the calibration of the spectrometer global momentum scale. The NA62 preliminary measurement of the $\pi^{0}$ TFF slope parameter is:

$$
a=\left(3.70 \pm 0.53_{\text {stat }} \pm 0.36_{\text {syst }}\right) \times 10^{-2}
$$

This measurement represents the first $5.8 \sigma$ observation of a positive $\pi^{0}$ electromagnetic TFF slope in the time-like region of the momentum transfer. The best fit result is shown in figure 1 together with the comparison with previous measurements from $\pi^{0}$ Dalitz decays. The NA62 result improves the precision of previous measurement by an order of magnitude, and is consistent with theoretical predictions.

\section{Measurement of the $K^{+} \rightarrow \pi^{+} v \bar{v}$ branching ratio}

The Flavour Changing Neutral Current $K \rightarrow \pi v \bar{v}$ decays proceed through box and electroweak penguin diagrams and are heavily suppressed in the Standard Model (SM). Using the values of the Cabibbo-Kobayashi-Maskawa (CKM) matrix elements as external inputs, the Standard Model predicts[21, 22]:

$$
\begin{aligned}
\operatorname{BR}_{\mathrm{SM}}\left(K^{+} \rightarrow \pi^{+} v \bar{v}\right) & =(8.4 \pm 1.0) \cdot 10^{-11} \\
\operatorname{BR}_{\mathrm{SM}}\left(K_{L} \rightarrow \pi^{0} v \bar{v}\right) & =(3.4 \pm 0.6) \cdot 10^{-11}
\end{aligned}
$$

where the uncertainties are dominated by the experimental knowledge of the external inputs.

The $K \rightarrow \pi v \bar{v}$ decays are very sensitive to physics beyond the SM. New particles at mass scales up to $100 \mathrm{TeV}$ are expected to produce significant variations of the $K \rightarrow \pi v \bar{v}$ branching ratios from SM predictions, which could be detected with a $10 \%$ precise measurement.

Only the charged mode has been observed so far. The present status of BR measurements is[2325]:

$$
\begin{aligned}
& \operatorname{BR}\left(K^{+} \rightarrow \pi^{+} v \bar{v}\right)=\left(17.3_{-10.5}^{+11.5}\right) \times 10^{-11} \\
& \operatorname{BR}\left(K_{L} \rightarrow \pi^{0} v \bar{v}\right)<2.6 \times 10^{-8} \quad(90 \% \text { C.L. })
\end{aligned}
$$




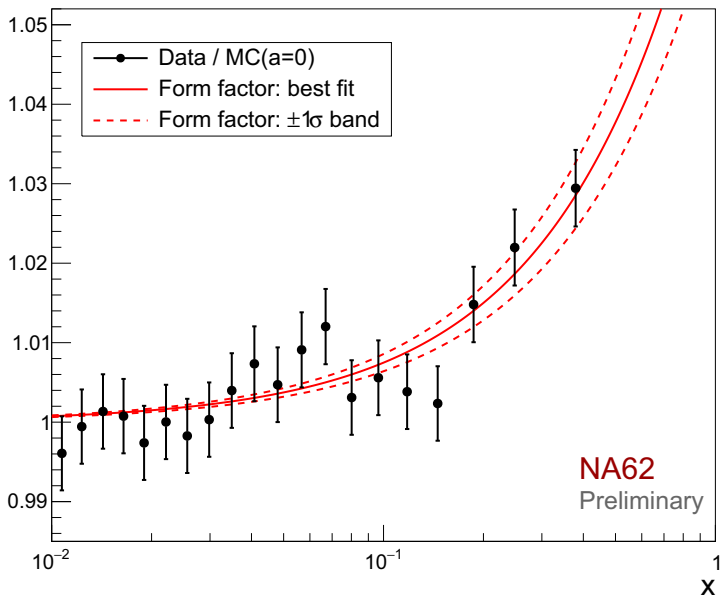

$\pi^{0}$ TFF Slope Measurements from $\pi_{\mathrm{D}}^{0}$

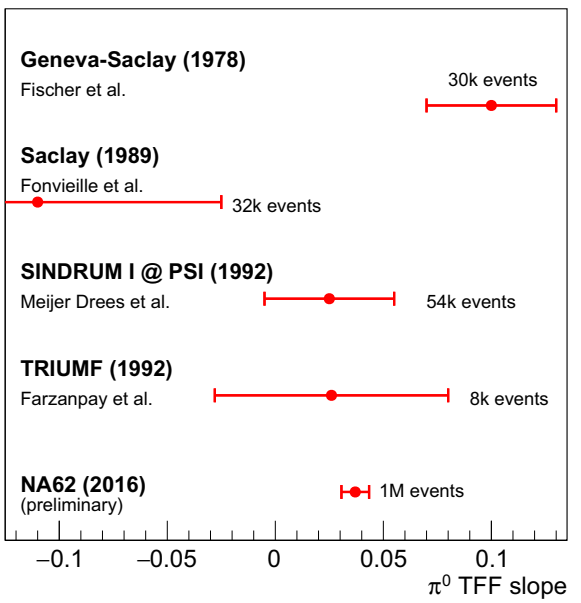

Figure 1. Measurement of the $\pi^{0}$ TFF slope parameter $a$. (left) Fit result showing the ratio data/MC as a function of the $x$ variable, with the MC sample weighted to obtain a flat TFF $(a=0)$. The events are divided into 20 equipopulous bins. The solid line represents the squared TFF function $|\mathcal{F}(x)|^{2}$ with a value of the slope parameter $a$ equal to the fit central value. Dashed lines indicate the $1 \sigma$ band. (right) Comparison of this result with previous experiments measuring the TFF slope from $\pi_{D}^{0}$ decays.

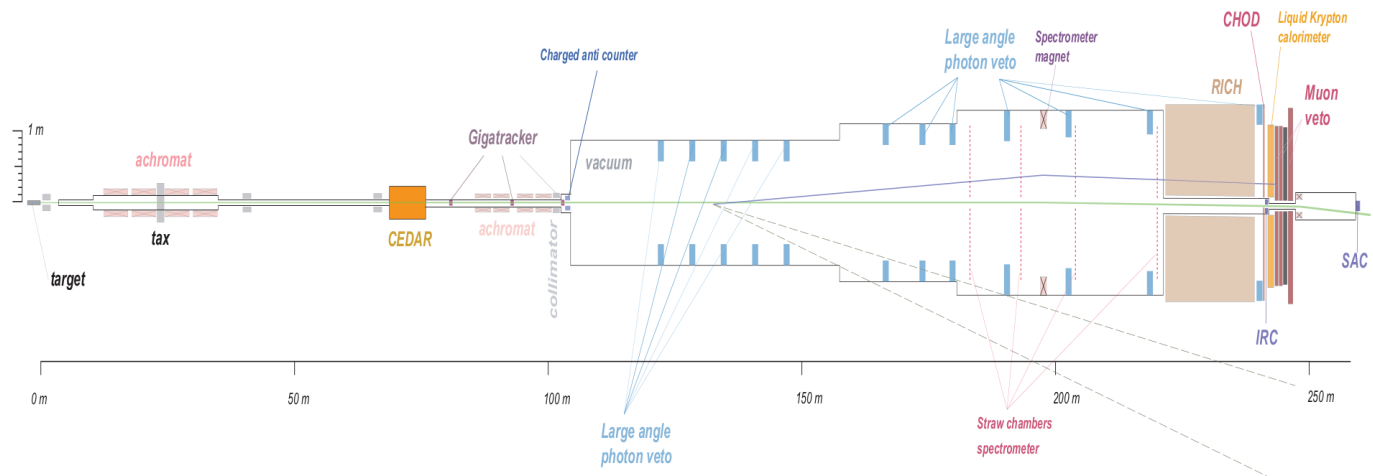

Figure 2. The NA62 layout for $K^{+} \rightarrow \pi^{+} \nu \bar{v}$ measurement.

\subsection{The NA62 experimental apparatus for $K^{+} \rightarrow \pi^{+} v \bar{v}$}

The main goal of the NA62 experiment at CERN SPS[26, 27] is to measure the $K^{+} \rightarrow \pi^{+} v \bar{v}$ branching ratio with a precision of $10 \%$ or better. The experiment plans to collect about $10^{13} \mathrm{kaon}$ in-flight decays in a few years, with a $10 \%$ acceptance. The extremely small branching ratio of the $\mathrm{BR}\left(\mathrm{K}^{+} \rightarrow\right.$ $\left.\pi^{+} v \bar{v}\right)$ decay combined with the weak signature of the signal events requires a very challenging $O\left(10^{12}\right)$ suppression of the main $K^{+}$decay modes. Several independent experimental techniques are required to reach this level of background suppression. 
The NA62 apparatus for the $K^{+} \rightarrow \pi^{+} v \bar{v}$ measurement, developed and built between 2008 and 2014 and replacing the NA48 one, is sketched in figure 2. The primary $400 \mathrm{GeV} / \mathrm{c}$ proton beam from CERN SPS impinges on a Be target producing secondary hadrons. A $100 \mathrm{~m}$ long beam line selects, collimates, focuses and transports positively charged particles of $(75 \pm 0.8) \mathrm{GeV} / \mathrm{c}$ momentum down to a $100 \mathrm{~m}$ long evacuated decay region. Kaons provide about $6 \%$ of the $750 \mathrm{MHz}$ particle rate in the charged beam. They are identified and timestamped by a nitrogen-filled Čerenkov counter (KTAG) located on the beam line. A beam spectrometer with three silicon pixel stations (GTK) of $6 \times 3 \mathrm{~cm}^{2}$ surface traces and timestamps all the beam particles upstream of the evacuated decay region. A guard ring detector (CHANTI) at the entrance of the decay volume tags upstream hadronic interactions. About $10 \%$ of beam kaons decay in the vacuum region between the beam line and the downstream detectors. Large angle annular lead glass electromagnetic calorimeters (LAV) surround the decay and downstream volumes to detect $\gamma$ up to $50 \mathrm{mrad}$. Charged particles are traced by a magnetic spectrometer with four straw tubes stations in vacuum, located at the downstream end of the decay volume. Holes around the beam axis in the spectrometer chambers allow undecayed particles to pass through without interacting. The large vacuum volume ends at the last spectrometer station and downstream the beam passes in vacuum inside a beam pipe.

A $17 \mathrm{~m}$ long neon-filled RICH counter provides particle identification for $\pi^{+}, \mu^{+}$and $e^{+}$up to $40 \mathrm{GeV} / \mathrm{c}$. The time of charged particles is measured both with the RICH and with two scintillator hodoscope planes (CHOD). The NA48 liquid kripton eletromagnetic calorimeter (LKr) detects forward $\gamma$ and contributes to charged particle identification. A shashlik small-angle annular calorimeter (IRC) in front of $\mathrm{LKr}$ efficiently detects $\gamma$ which would otherwise interact with the LKr inner edge outside the $\mathrm{LKr}$ acceptance. An hadronic calorimeter made by two modules of iron-scintillator sandwiches (MUV1 and MUV2) provides further $\pi^{+} / \mu^{+}$separation and hadronic energy based triggers. A fast scintillator array (MUV3) downstream of the calorimeters identifies and triggers muons with sub-nanosecond time resolution. In order to detect $\gamma$ down to zero angle a shashlik calorimeter (SAC) is located further downstream on the beam line after a dipole magnet deflecting undecayed charged particles outside its acceptance. All the downstream detectors see less than $1 \%$ of the beam rate.

A multi-level trigger architecture is used. The level zero trigger is based on timing information from $\mathrm{CHOD}, \mathrm{RICH}$ and MUV3 and on calorimetric variables from electromagnetic and hadronic calorimeters, determined using FPGA mounted on the TEL62 readout boards [28]. Data from KTAG, LAV and magnetic spectrometer are used in the software-based higher level trigger.

NA62 collected data in 2014 and 2015. The hardware and readout of the detectors have been commissioned at $10 \%$ of the nominal beam intensity, the beam line up to the nominal intensity. The GTK ran with a partial hardware configuration. Data samples with beam intensity varying from percent of the nominal up to the nominal one have been recorded in 2015. Data at low intensity have been triggered with CHOD only. At higher intensities $K^{+} \rightarrow \pi^{+} v \bar{v}$-like events have been triggered at level zero by calorimetric information. A preliminary analysis of a subset of the low intensity sample, aiming to exploit the data quality in view of the $K^{+} \rightarrow \pi^{+} v \bar{v}$ measurement, is described in the following sections. The analysis of the full 2015 data set is in progress.

\subsection{Principles of the measurement}

The $K^{+} \rightarrow \pi^{+} v \bar{v}$ signature in NA62 consists of a track in the GTK compatible with a kaon hypothesis, a charged particle in the detectors downstream and nothing else. Other (more abundant) kaon decays and beam related activity are the main sources of background.

For each event we define the squared missing mass as $m_{\text {miss }}=\left(P_{K}-P_{\pi}\right)^{2}$, where $P_{K}$ is the 4momentum of the beam kaon and $P_{\pi}$ that of the downstream track under pion mass hypothesis. As can be seen in figure 3 , the expected squared missing mass distribution of the signal has a broad shape 

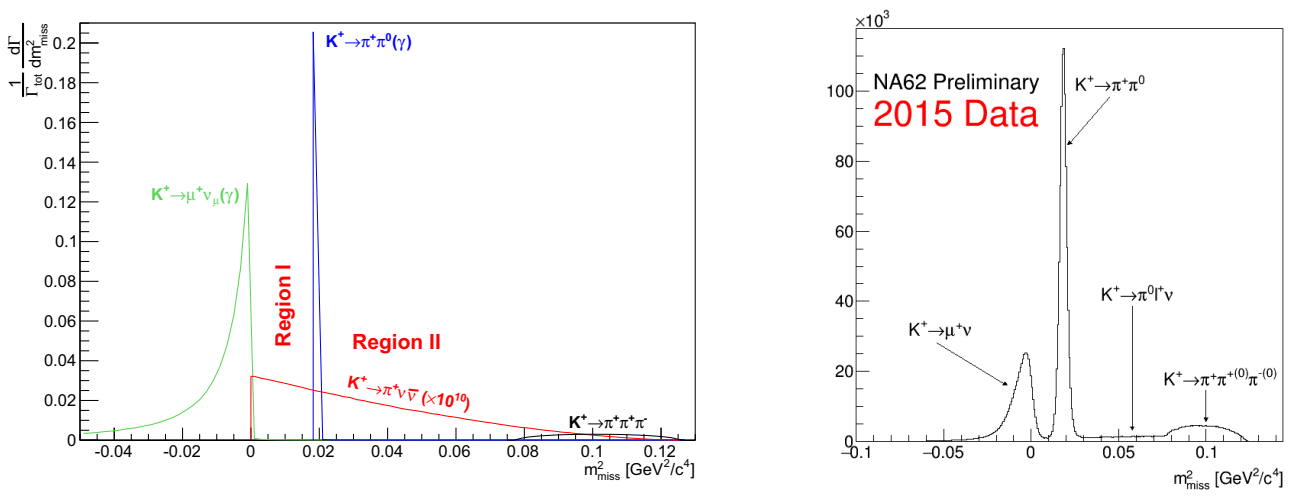

Figure 3. (left) Squared missing mass distribution expected for $K^{+} \rightarrow \pi^{+} v \bar{v}$ signal (multiplied by $10^{10}$ ) and for main kaon decays $K^{+} \rightarrow \mu^{+} v_{\mu}\left(K_{\mu 2}\right), K^{+} \rightarrow \pi^{+} \pi^{0}\left(K_{2 \pi}\right)$ and $K^{+} \rightarrow \pi^{+} \pi^{+} \pi^{-}\left(K_{3 \pi}\right)$. (right) Squared missing mass distribution measured on single-track selected events collected in 2015.

typical of three-body decays, while more than $90 \%$ of the charged kaon decays are peaking. Two signal regions are defined outside the $K^{+} \rightarrow \pi^{+} \pi^{0}$ and $K^{+} \rightarrow \mu^{+} v_{\mu}$ peaks. Semileptonic decays, radiative processes, beam induced tracks and reconstruction tails of main $K^{+}$decays are expected sources of background in these regions. Precise kinematic reconstruction, efficient photon detection, particle identification and sub-nanosecond timing coincidences between subdetectors must be employed to reach the required background rejection.

\subsection{Data quality analysis for the $K^{+} \rightarrow \pi^{+} v \bar{v}$ measurement}

The quality of data for $K^{+} \rightarrow \pi^{+} v \bar{v}$ measurement is studied after applying the following selection. Tracks reconstructed in the straw spectrometer are selected, matching in space CHOD signals and energy depositions in calorimeters. The event is required to contain a single track, i.e. a track not forming a vertex with any other in-time track in the decay region between the last GTK station and the first plane of the straw spectrometer. A vertex is defined as the average position of two tracks projected back in the decay region at their closest approach, provided their distance (CDA) is less than $1.5 \mathrm{~cm}$. In order to select a single track originating from a kaon decay, a GTK track is required to match the downstream track both in time and space, forming a vertex in the decay region with it, and also to be in time with a kaon-like signal in KTAG. The time resolution of RICH, KTAG, GTK track, and CHOD have been measured in the range between 70 and $200 \mathrm{ps,} \mathrm{matching} \mathrm{the} \mathrm{design} \mathrm{values.}$ Figure 4(left) shows the distribution of events in the track momentum-squared missing mass plane for 2015 data recorded at low intensity. Regions populated by main $K^{+}$decays are clearly visible. The same distribution for events not related to kaons, selected with KTAG in anti-coincidence, is plotted in figure 4(right). The analysis of these events show that beam $\pi^{+}$decays, elastic scattering of beam particles in the material along the beam line and inelastic scatterings in the last GTK station are the main sources of downstream tracks from beam-related activity. Kinematic resolution, particle identification and $\gamma$ rejection are studied using the sample of single track events from kaon decays selected above.

The resolution on $m_{\text {miss }}^{2}$ measured from the width of the $K^{+} \rightarrow \pi^{+} \pi^{0}$ peak is about $1.2 \times$ $10^{-3} \mathrm{GeV}^{2} / \mathrm{c}^{4}$, close to the $10^{-3} \mathrm{GeV}^{2} / \mathrm{c}^{4}$ design value. Taking the average beam momentum in 

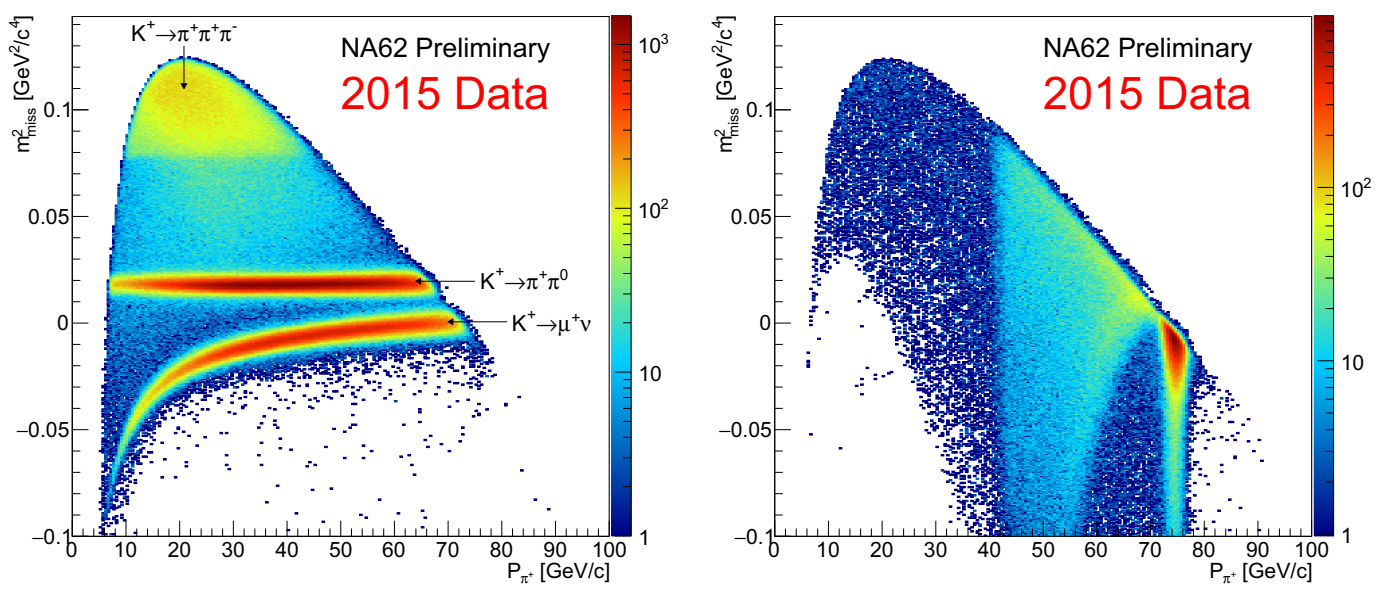

Figure 4. Squared missing mass ditribution vs. pion momentum, for: kaon-related events selected with KTAG in coincidence (left), non-kaon-related events with KTAG in anti-coincidence (right).

place of the event-by-event kaon momentum measured with the Gigatracker increases the resolution by a factor 3. The $m_{\text {miss }}^{2}$ variable obtained from NA62 spectrometers is used to reject $K^{+} \rightarrow \pi^{+} \pi^{0}$ and $K^{+} \rightarrow \mu^{+} v$ events. The measured suppression for $K^{+} \rightarrow \pi^{+} \pi^{0}$ is of the order of $10^{3}$, still below the design value $\left(10^{4}-10^{5}\right)$. This is due to $m_{\text {miss }}^{2}$ tails caused by beam track mis-reconstruction in the still partially equipped GTK.

The NA62 particle identification, based on a combined usage of RICH and calorimeters, is designed to separate $\pi^{+}$from $\mu^{+}$and $e^{+}$and reach a $10^{7}$ suppression of $K^{+} \rightarrow \mu^{+} v$ in addition to the kinematic rejection. Pure samples of $K^{+} \rightarrow \pi^{+} \pi^{0}$ and $K^{+} \rightarrow \mu^{+} v$ events are used to study the $\pi^{+} / \mu^{+}$ separation in RICH and calorimeters. A $10^{2}$ muon suppression with $80 \%$ pion efficiency is obtained by the RICH in the required track momentum region $(15 \div 35) \mathrm{GeV} / \mathrm{c}$, as shown in figure 5 . A simple cut-based analysis of calorimeters data provide an additional muon suppression factor between $10^{4}$ and $10^{6}$ for a pion efficiency ranging between $90 \%$ and $50 \%$. More efficient analysis techniques are under study.

The NA62 layout is designed to provide a $10^{8}$ suppression for $K^{+} \rightarrow \pi^{+} \pi^{0}$ events in addition to the kinematic rejection by detecting at least one photon from $\pi^{0}$ decay in one of the electromagnetic calorimeters $\mathrm{LAV}, \mathrm{LKr}, \mathrm{IRC}$ and SAC, covering an overall angular region between 0 and $50 \mathrm{mrad}$. The suppression of $\pi^{0}$ s from $K^{+} \rightarrow \pi^{+} \pi^{0}$ decays is measured on kinematically selected $K^{+} \rightarrow \pi^{+} \pi^{0}$ events. The measurement of $\pi^{0}$ suppression is statistically limited at a lower limit of $\approx 10^{6}$.

Commissioning of NA62 for $K^{+} \rightarrow \pi^{+} v \bar{v}$ is almost completed. The preliminary data quality analysis of low intensity 2015 data shows that NA62 is approaching the $K^{+} \rightarrow \pi^{+} \nu \bar{v}$ design sensitivity.

\section{References}

[1] V. Fanti et al. (NA48 Collaboration), Nucl. Instrum. Methods A 574, 433 (2007).

[2] C. Lazzeroni et al. (NA62 Collaboration), Phys. Lett. B 719, 326 (2013).

[3] M. Gell-Mann and F. Zachariasen, Phys. Rev. 124, 953 (1961). 

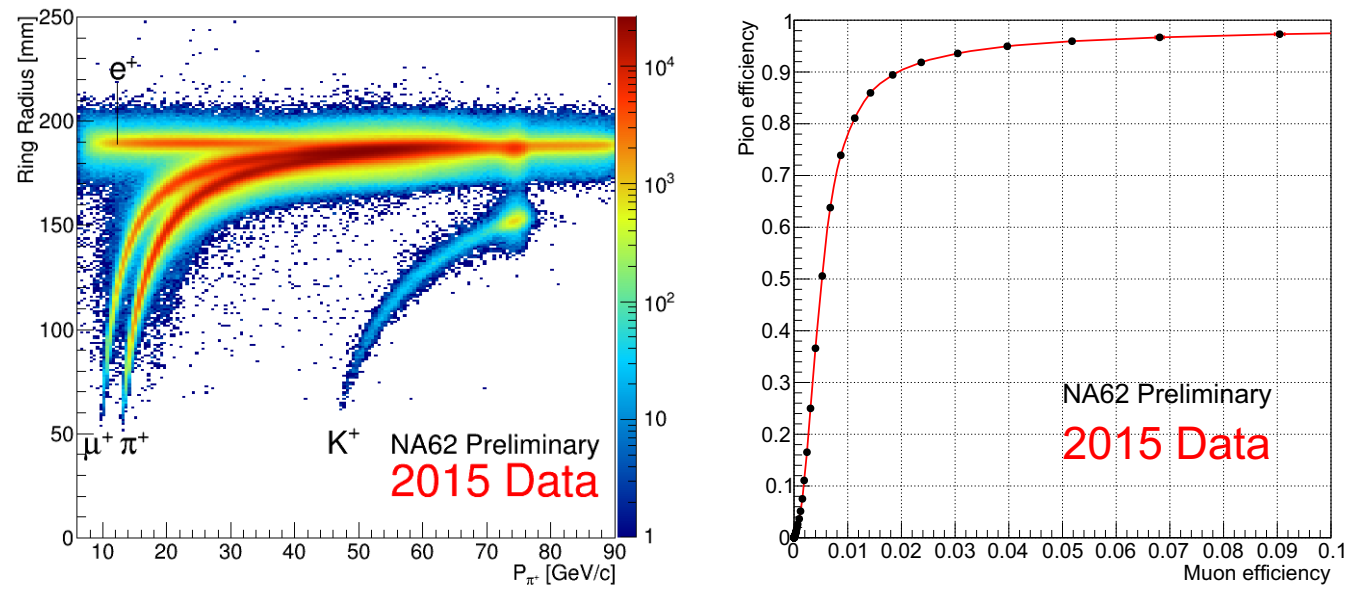

Figure 5. Particle identification with NA62 RICH: (left) RICH ring radius vs. momentum; (right) Pion efficiency vs. muon suppression (efficiency), for particle momentum between 15 and $35 \mathrm{GeV} / \mathrm{c}$.

[4] P. Lichard, Phys. Rev. D 83, 037503 (2011).

[5] K. Kampf et al., Eur. Phys. J. C 46, 191 (2006).

[6] T. Husek and S. Leupold, Eur. Phys. J. C 75, 586 (2015).

[7] P. Masjuan, Phys. Rev. D 86, 094021 (2012).

[8] M. Hoferichter et al., Eur. Pjys. J. C 74, 3180 (2014).

[9] J. Fisher et al.(Geneva-Saclay Collaboration), Phys. Lett. B 73, 359 (1978).

[10] H. Fonvieille et al., Phys. Lett. B 233, 65 (1989).

[11] H. Forzanpay et al., Phys. Lett. B 278, 413 (1992).

[12] R. Meijer Drees et al.(SINDRUM Collaboration), Phys. Rev. D 45, 1439 (1992).

[13] H.J. Berend et al.(CELLO Collaboration), Zeit. Phys. C 49, 401 (1991).

[14] et al.(CLEO Collaboration), Phys. Rev. D 57, 33 (1998).

[15] et al.(BaBar Collaboration), Phys. Rev. D 80, 052002 (2009).

[16] et al.(Belle Collaboration), Phys. Rev. D 86, 092007 (2012).

[17] D.W. Joseh, Il Nuovo Cimento 16, 997 (1960).

[18] B.E. Lautrup and J. Smith, Phys. Rev. D 3, 1122 (1971).

[19] M. Mikaelian and J. Smith, Phys. Rev. D 5, 1763 (1972).

[20] T. Husek et al., Phys. Rev. D 92, 054027 (2015).

[21] J. Brod et al., Phys. Rev. D 83, 034030 (2011).

[22] A.J. Buras et al., JHEP 11, 033 (2015).

[23] S. Adler et al. (E787 Collaborations), Phys. Rev. D 77, 052003 (2008).

[24] A.V Artamonov et al. (E949 Collaborations), Phys. Rev. D 79, 092004 (2009).

[25] J.K. Ahn et al. (E381a Collaboration), Phys. Rev. D 81, 072004 (2010).

[26] G. Anelli et al., CERN-SPSC-2005-013; SPSC-P-326.

[27] NA62 Technical Design Document, NA62-10-07.

[28] B. Angelucci et al., JINST 7, C02046 (2012). 